\title{
HOMOMORPHISMS OF RINGS OF GERMS OF ANALYTIC FUNCTIONS
}

\author{
WILLIAM R. ZAME
}

\begin{abstract}
Let $S$ and $S^{\prime}$ be complex analytic manifolds with $S$ Stein. Let $X \subset S$ and $X^{\prime} \subset S^{\prime}$ be compact sets with $X$ holomorphically convex. Denote by $\mathcal{C}(X)$ (respectively $\mathcal{O}\left(X^{\prime}\right)$ ) the ring of germs on $X$ (respectively $X^{\prime}$ ) of functions analytic near $X$ (respectively $X^{\prime}$ ). It is shown that each nonzero homomorphism of $\mathcal{C}(X)$ into $\mathcal{O}\left(X^{\prime}\right)$ is given by composition with an analytic map defined in a neighborhood of $X^{\prime}$ and taking values in $S$.
\end{abstract}

If $S$ and $S^{\prime}$ are complex analytic manifolds, then every analytic mapping of $S$ into $S^{\prime}$ induces (via composition) a homomorphism of the ring of analytic functions on $S^{\prime}$ into the ring of analytic functions on $S$. It is an important and deep result that the converse is also true, providing that $S$ is a Stein manifold (see [1]). In this note we obtain an analogous result for homomorphisms of rings of germs of analytic functions on compact subsets of a complex analytic manifold. We show that each such homomorphism is given by composition with an analytic mapping.

1. Preliminaries and notation. Let $S$ be a Stein manifold and $U$ an open subset of $S$. We denote by $\mathcal{O}(U)$ the ring of analytic functions on $U$. It is well known (see [1], for example) that each nonzero complex-valued homomorphism of $\mathcal{O}(U)$ is continuous with respect to the topology on $\mathcal{O}(U)$ of uniform convergence on compact subsets of $U$. We denote the space of such homomorphisms by $\Delta \mathcal{O}(U)$ If $f \in \mathcal{O}(U)$ then $f^{\wedge}$ denotes the function on $\Delta \mathcal{O}(U)$ defined by $f^{\wedge}(\alpha)=\alpha(f)$ for each $\alpha$ in $\Delta \mathcal{O}(U)$.

Since $S$ is Stein, $\Delta \mathcal{O}(S)=S$, so we have the natural restriction map $\pi_{U}: \Delta \mathcal{O}(U) \rightarrow S$ given by $\pi_{U^{-}}(\alpha)(f)=\alpha(f \mid U)$. Rossi [4] has shown that $\Delta \mathcal{O}(U)$ admits the structure of a Stein manifold in such a way that: (i) the evaluation map $U \rightarrow \Delta \mathcal{C}(U)$ is a biholomorphism of $U$ with an open subset of $\Delta \mathcal{O}(U)$ (we will regard $U$ as an open subset of $\Delta O(U)$ ); (ii) if $f \in \mathcal{O}(U)$ then $f^{\wedge}$ is the unique analytic extension of $f$ to $\Delta \mathcal{O}(U)$ (so that $\mathcal{O}(\Delta \mathcal{O}(U))=\mathcal{O}(U))$; (iii) $\pi_{U}$ is locally a biholomorphism.

Received by the editors September 2, 1971.

AMS 1970 subject classifications. Primary 32E25, 46E25.

Key words and phrases. Germs of analytic functions, holomorphically convex sets.

${ }^{1}$ Supported by National Science Foundation Grant GP-19011. 
If $X$ is a compact subset of $S$, we denote by $\mathcal{C}(X)$ the ring of germs on $X$ of functions analytic near $X$. If $f$ is analytic in a neighborhood of $X$, we denote its germ on $X$ by $f ; f$ is called a representative of $f$. We will also regard a germ in $\mathcal{C}(X)$ as a continuous function on $X$. It was shown in [2] and [6] that each nonzero complex-valued homomorphism on $\mathcal{O}(X)$ is continuous, relative to the natural inductive limit topology on $\mathcal{O}(X)$. We say that $X$ is holomorphically convex if each such homomorphism is given by evaluation at a point of $X$. Equivalently, $X$ is holomorphically convex if and only if $\left\{\pi_{U}(\Delta \mathcal{O}(U)) ; U\right.$ is open and $\left.X \subset U\right\}$ is a fundamental system of neighborhoods of $X$. More information about holomorphically convex sets may be found in [2] and [5]. We refer to [1] for general information about several complex variables.

2. Main result. Now let $S$ and $S^{\prime}$ be complex analytic manifolds with $S$ Stein, and let $X \subset S$ and $X^{i} \subset S^{\prime}$ be compact sets with $X$ holomorphically convex. We wish to study homomorphisms of $\mathcal{O}(X)$ into $\mathcal{C}\left(X^{\prime \prime}\right)$. If $F$ is an analytic map of a neighborhood $U^{\prime}$ of $X^{\prime}$ into $S$ such that $F\left(X^{\prime}\right) \subset X$, then $F$ induces a homomorphism $\Phi_{F}: \mathcal{O}(X) \rightarrow \mathcal{O}\left(X^{\prime}\right)$ as follows. Let $f$ be in $O(X)$. Choose an open set $U$ containing $X$ and a represertative $f$ of $f$ which is analytic on $U$. Then $f \circ F$ is analytic on a neighborhood of $X^{\prime}$; we let $\Phi_{F}(f)$ be the germ of $f \circ F$ on $X^{\prime}$. By a straightforward calculation, we may verify that $\Phi_{F}$ is a well-defined homomorphism of $\mathcal{O}(X)$ into $\mathcal{O}\left(X^{\prime}\right)$. Our main result is that every homomorphism arises in this way.

THEOREM. Let $\Phi: \mathcal{C}(X) \rightarrow \mathcal{C}\left(X^{\prime}\right)$ be a nonzero homomorpiaism. Then there is an open set $U^{\prime}$ containing $X^{\prime}$ and an analytic function $F: U^{\prime} \rightarrow S$ such that $F\left(X^{\prime \prime}\right) \subset X$ and $\Phi=\Phi_{F}$. The germ of $F$ on $X^{\prime}$ is unique.

For the proof of this theorem we shall make use of two lemmas. If $T$ is a subset of $S$ and $\mathscr{F}$ is a subset of $\mathscr{O}(S)$, we say that $\mathscr{F}$ separates points on $T$ if for each $x$ and $y$ in $T$ with $x \neq y$ there is a function $f \in \mathscr{F}$ such that $f(x) \neq f(y)$. If the (complex) dimension of $S$ is $n$, we say that $\mathscr{F}$ prevides local coordinates on $T$ if for each $x$ in $T$ there are $n$ functions $f_{1}, \cdots, f_{n}$ in $\mathscr{F}$ such that $d f_{1} \wedge \cdots \wedge d f_{n}(x) \neq 0$.

Lemma 1. Let $\left\{f_{1}, \cdots, f_{k}\right\}$ be a subset of $\mathcal{C}(S)$ which separates points and provides local coordinates on $X$, and let $U$ be an open set containing $X$. Then there is an open sei $W$ containing $X$ such that for each $x$ in $X$, each integer $M$ and each $f$ in $C(U)$ vanishing to total order at least $M$ at $x$, there are functions $g_{1}, \cdots, g_{N}$ in $\mathcal{O}(W)$ and monomials $h_{1}, \cdots, h_{N}$ of degree $M$ in $f_{1}-f_{1}(x), \cdots, f_{k}-f_{k}(x)$ such that $f=\sum g_{i} h_{i}$ in $W$.

Proof. A straightforward compactness argument shows that there is an cpen set $U_{1}$ containing $X$ such that $\left\{f_{1}, \cdots, f_{k}\right\}$ separates points and provides local cocrdinates on $U_{1}$. Since $X$ is holomorphically convex, there is 
an open set $W$ containing $X$ such that $\pi_{W}(\Delta \mathcal{O}(W)) \subset U \cap U_{1}$. Let $x$ be a point of $X, M$ an integer, and $h_{1}, \cdots, h_{N}$ the monomials of degree $M$ in $f_{1}-f_{1}(x), \cdots, f_{k}-f_{\kappa}(x)$.

Let $\mathcal{O}$ be the sheaf of germs of analytic functions on $\Delta \mathcal{O}(W)$ and $\mathcal{O}^{\mathrm{V}}$ the $N$-fold Cartesian product. Let $\mathscr{I}$ be the ideal sheaf of the discrete variety $\pi_{W}^{-1}(x)$ and $\mathscr{I}_{1 I}$ the sheaf of ideals generated by $M$-fold products of elements of $\mathscr{I}$. For each $i$, let $\phi_{i}=\left(h_{i} \mid W\right)^{\wedge}$ and let $\mu: \mathcal{O}^{N} \rightarrow \mathscr{I}_{M}$ be given by $\mu\left(\gamma_{1}, \cdots, \gamma_{N}\right)=\sum \phi_{i} \gamma_{i}$. Observe that the functions

$$
\left(\left(f_{1}-f_{1}(x)\right) \mid W\right)^{\wedge}, \cdots,\left(\left(f_{k}-f_{k}(x)\right) \mid W\right)^{\wedge}
$$

provide local coordinates on $\Delta \mathcal{O}(W)$ and vanish simultaneously only on $\pi_{W}^{-1}(x)$. Thus at each point of $\pi_{W}^{-1}(x)$, each germ in $\mathscr{I}_{1 I}$ can be expressed locally as a power series in these functions, while at each point not in $\pi_{W}^{-1}(x)$ at least one of these functions is locally a unit. It follows that $\mu$ is surjective.

This gives rise to the following short exact sequence of sheaves over $\Delta \mathcal{O}(W)$ :

$$
0 \rightarrow \operatorname{ker} \mu \rightarrow \mathcal{O}^{\mathrm{V}} \rightarrow \mathscr{I}_{M} \rightarrow 0 \text {. }
$$

This induces a long exact cohomology sequence, the relevant terms of which are:

$$
H^{0}\left(\Delta \mathcal{O}(W), \mathcal{O}^{. v}\right) \stackrel{\mu^{*}}{\rightarrow} H^{0}\left(\Delta \mathcal{O}(W), \mathscr{I}_{.1}\right) \rightarrow H^{1}(\Delta \mathcal{O}(W) \text {, ker } \mu) .
$$

Since $\Delta \mathcal{C}(W)$ is a Stein manifold and $\operatorname{ker} \mu$ is a sheaf of relations on $\Delta \mathcal{C}(W)$, it follows from Cartan's Theorem B that $H^{1}(\Delta \mathcal{O}(W), \operatorname{ker} \mu)=0$, so that $\mu^{*}$ is surjective.

Finally, if $f \in \mathcal{O}(U)$ vanishes to total order at least $M$ at $x$, then $(f \mid W)^{\wedge}=$ $f \circ \pi_{W}$ vanishes to total order at least $M$ at each point of $\pi_{W}^{-1}(x)$. Thus $(f \mid W)^{\wedge} \in H^{0}\left(\Delta O(W), \mathscr{I}_{. I}\right)$. Thus $(f \mid W)^{\wedge}$ is the image under $\mu^{*}$ of an $N$-tuple of functions in $\mathcal{O}(\Delta \mathcal{O}(W))=\mathcal{O}(W)$, which is the desired result.

LEMMA 2. Let $\left\{f_{1}, \cdots, f_{k}\right\}$ be a subset of $\mathcal{O}(S)$ which separates points and provides local coordinates on $X$. If $\Phi_{1}$ and $\Phi_{2}$ are nonzero homomorphisms of $\mathcal{C}(X)$ into $\mathcal{C}\left(X^{\prime}\right)$ such that $\Phi_{1}\left(f_{i}\right)=\Phi_{2}\left(f_{i}\right)$ for each $i$, then $\Phi_{1}=\Phi_{2}$.

Proof. Since every nontrivial homomorphism of $\mathcal{C}(X)$ into $C$ is given by evaluation at a point of $X$, we can define maps $\Phi_{j}^{*}: X^{\prime} \rightarrow X$ by requiring that for each $f \in \mathcal{O}(X)$ and $y \in X^{\prime}, \Phi_{j}^{*}(y)(f)=\Phi_{j}(f)(y)$ for $j=1,2$. For each $i$ and each $y \in X^{\prime}$ we have:

$$
f_{i}\left(\Phi_{1}^{*}(y)\right)=\Phi_{1}\left(f_{i}\right)(y)=\Phi_{2}\left(f_{i}\right)(y)=f_{i}\left(\Phi_{2}^{*}(y)\right) .
$$

Since $\left\{f_{1}, \cdots, f_{k}\right\}$ separates points on $X$, it follows that $\Phi_{1}^{*}=\Phi_{2}^{*}$. 
Let $f$ be in $\mathcal{C}(X)$. In order to show that $\Phi_{1}(f)=\Phi_{2}(f)$ it suffices to show that $\Phi_{1}(f)-\Phi_{2}(f)$ vanishes to arbitrarily high order at each point of $X^{\prime}$. To this end, let $M$ be an intege: and $y$ a point of $X^{\prime}$; set $x=\Phi_{1}^{*}(y)=$ $\Phi_{2}^{*}(y)$. In a neighborhood of $x$, some representative of $f$ may be represented by a power series in $f_{1}-f_{1}(x), \cdots, f_{k}-f_{k}(x)$; let $P_{M}$ denote the sum of the terms of this series whose total order in $f_{1}-f_{1}(x), \cdots, f_{k}-f_{k}(x)$ does not exceed $M-1$. Hence the germ $\boldsymbol{f}-\boldsymbol{P}_{M}$ vanishes to total order at least $M$ at $x$. In view of Lemma 1 , we may find germs $g_{1}, \cdots, g_{N}$ in $\mathcal{O}(X)$ and monomials $h_{1}, \cdots, h_{Y}$ of order $M$ in $f_{1}-f_{1}(x), \cdots, f_{k}-f_{k}(x)$ such that $\boldsymbol{f}-\boldsymbol{P}_{\lambda}=\Sigma \boldsymbol{g}_{i} \boldsymbol{h}_{i}$.

Since $\Phi_{1}$ and $\Phi_{2}$ are nonzero, it follows that $\Phi_{1}(\mathbf{1})=\Phi_{2}(\mathbf{1})=1$, so that $\Phi_{1}\left(\boldsymbol{P}_{M}\right)=\Phi_{2}\left(\boldsymbol{P}_{\lambda I}\right)$. Hence

$$
\begin{aligned}
\Phi_{1}(f)-\Phi_{2}(f) & =\Phi_{1}\left(f-P_{M}\right)-\Phi_{2}\left(f-P_{M}\right) \\
& =\sum \Phi_{1}\left(h_{j}\right)\left\{\Phi_{1}\left(g_{j}\right)-\Phi_{2}\left(g_{j}\right)\right\}
\end{aligned}
$$

since $\Phi_{1}\left(h_{j}\right)=\Phi_{2}\left(h_{j}\right)$. Since $h_{j}$ is the product of $M$ germs that vanish at $x$, $\Phi_{1}\left(h_{j}\right)$ is the product of $M$ germs that vanish at $y$. It follows that $\Phi_{1}(f)-$ $\Phi_{2}(f)$ vanishes to order at ieast $M$ at $y$. This completes the proof.

Proof of THE Theorem. By the imbedding theorem for Stein manifolds, we can find functions $g_{1}, \cdots, g_{k}$ in $\mathcal{C}(S)$ such that $G=\left(g_{1}, \cdots, g_{k}\right)$ is a regular proper imbedding of $S$ as a closed submanifold of $C^{h}$. We can find an open set $\Omega$ containing $G(S)$ and a holomorphic retraction $h: \Omega \rightarrow G(S)$. If $f$ is analytic in an open set containing $G(X)$, then $f \circ G$ is analytic in an open set containing $X$, so we have defined a homomorphism $v: \mathcal{O}(G(X)) \rightarrow \mathcal{O}(X)$. Observe that $v(f)=v(g)$ whenever $f$ and $g$ have representatives that agree in a $G(S)$-neighborhood of $G(X)$. Leî $\Phi^{\prime}=\Phi \circ v$ and set $\varphi_{j}=\Phi^{\prime}\left(z_{j}\right)=\Phi_{(}\left(g_{j}\right)$.

We can find an open set $U^{\prime}$ containing $X^{\prime}$ and functions $\varphi_{1}, \cdots, \varphi_{k}$ in $\mathcal{O}\left(U^{\prime}\right)$ which represent $\varphi_{1}, \cdots, \varphi_{k}$. Set $\varphi=\left(\varphi_{1}, \cdots, \varphi_{k}\right): U^{\prime} \rightarrow C^{k}$ and consider the homomorphism $\Phi_{4}: \mathcal{O}(G(X)) \rightarrow \mathcal{O}\left(X^{\prime}\right)$. It is easy to see that $\Phi_{\varphi}\left(z_{i}\right)=\varphi_{i}$ for each $i$. Since $X$ is holomorphically convex in $S, G$ is an imbedding and $G(S)$ is closed in $C^{k}$, it follows that $G(X)$ is homomorphically convex in $C^{k}$ (see [5]). From Lemma 2 we see that $\Phi_{\varphi}=\Phi^{\prime}$.

Now set $F=G^{-1} \circ h^{\circ} \varnothing: U^{\prime} \rightarrow S$. If $f_{i}$ denotes the germ of $g_{i} \circ G^{-1} \circ h$ on $G(X)$, then we see that $\Phi^{\prime}\left(f_{i}\right)=\Phi^{\prime}\left(z_{i}\right)=\Phi\left(g_{i}\right)$. Hence $\Phi_{F}\left(g_{i}\right)=$ $\Phi^{\prime}\left(f_{i}\right)=\Phi\left(g_{i}\right)$. Using Lemma 2 again, it follows that $\Phi=\Phi_{F}$. (Observe that this argument shows that $\varphi\left(U^{\prime}\right) \subset G(S)$ so that $F=G^{-1} \circ h \circ \varphi=$ $G^{-1} \circ \varphi$.) It is easy to see that $F \mid X^{\prime}=\Phi^{*}$ so that $F\left(X^{\prime}\right) \subset X$. Finally, a straightforward calculation shows that $\Phi_{F}$ depends only on the germ of $F$ on $X^{\prime}$ and that functions representing different germs induce distinct homomorphisms. 
3. Remarks. It is tempting to try to generalize the theorem to the case in which $X$ is not holomorphically convex by passing to the space of nonzero homomorphisms of $\mathcal{O}(X)$ into $C$. In general, however, this space admits no natural imbedding into a Stein manifold (see [2] and [6]).

If $S^{\prime}$ is Stein and $X^{\prime}$ is holomorphically convex, then $\Phi=\Phi_{F}$ is an isomorphism if and only if $F$ is a biholomorphism of a neighborhood of $X^{\prime}$ with a neighborhood of $X$.

The author does not know whether the theorem remains true in the context of analytic spaces, rather than manifolds.

\section{REFERENCES}

1. R. C. Gunning and H. Rossi, Analytic functions of several complex variables, Prentice-Hall, Englewood Cliffs, N.J., 1965. MR 31 \#4927.

2. R. Harvey and R. O. Wells, Compact holomorphically convex subsets of a Stein manifold, Trans. Amer. Math. Soc. 136 (1969), 509-516. MR 38 \#3470.

3. H. Rossi, Holomorphically convex sets in several complex variables, Ann. of Math. (2) 74 (1961), 470-493. MR 24 \#A3310.

4. __, On envelopes of holomorphy, Comm. Pure Appl. Math. 16 (1963), 9-17. MR 26 \#6436.

5. $\mathrm{R}_{z} \mathrm{O}$. Wells, Jr., Holomorphic hulls and holomorphic convexity, Proc. Conference Complex Analysis (Rice University, Houston, Tex., 1967), Rice Univ. Studies 54 (1968), no. 4, 75-84. MR 39 \#3029.

6. W. R. Zame, Algebras of analytic germs (to appear).

Department of Mathematics, Rice University, Houston, TeXas 77001 